\title{
Neuroprotective role of PKR in a model of neurodegeneration due to mild impairment of oxidative metabolism
}

\author{
François Mouton-Liger ${ }^{1,2^{*}}$, Anne-Sophie Rebillat ${ }^{1}$, Sarah Gourmaud ${ }^{1}$, Audrey Leguen ${ }^{1}$, Claire Paquet ${ }^{1,2}$, \\ Laurent Pradier ${ }^{3}$, Thomas Rooney ${ }^{3}$, Jacques Hugon ${ }^{1,2}$ \\ From Molecular Neurodegeneration: Basic biology and disease pathways \\ Cannes, France. 10-12 September 2013
}

\section{Background}

Abnormalities in oxidative metabolism, inflammation, and selective neuronal death are described features of Alzheimer's disease (AD) pathology. Brain thiaminedependent enzymes play an important role in energy metabolism and display reduced activity in AD. Thiamine (vitamin B1) deficiency (TD) induces regionally selective neuronal death in animal and human brains. TD-induced neuronal loss is associated with a mild and chronic impairment of oxidative metabolism as well as inflammatory responses with cytokine production. These features make the TD model amenable to investigate the cellular mechanisms of neurodegeneration. PKR (double stranded-RNA dependent protein kinase) is activated by $A \beta_{1-42}$, and by various cellular stresses including oxidative stress. Once activated, PKR acts as a pro-apoptotic kinase and negatively controls the initial step of protein translation by the elF $2 \alpha$ phosphorylation, leading to an increase of BACE1 translation. In addition, it has been demonstrated that TD promotes PKR activation.

\section{Learning objective}

The goal of this study was to use the TD model in mice to assess in vivo the involvement of PKR in the neuronal death, inflammation and in the neuropathological mechanisms of AD. All results of this TD model are compared with those obtained in controls (saline injected).

\section{Methods}

C57BL/6J mice, housed in a controlled environment, have been fed either a control diet or a thiamine deficient diet ad libitum. TD animals also received a daily intraperitoneal injection of a thiamine antagonist, pyrithiamine hydrobromide for 10 days while control animals will be injected with saline.

\section{Results}

Our results showed that the PKR-elF2 $\alpha$ pathway, $\mathrm{BACE} 1$ levels and $\mathrm{A} \beta$ production are increased in the thalamus and cerebellum of the TD model. These findings are associated with microglial activation and neuronal death. Performance on the rotarod task also declines in the animal TD model in a highly reproducible manner.

\section{Conclusion}

Our findings suggest a role in vivo of the kinase PKR in neuronal degeneration linked to oxidative metabolism impairment.

\section{Authors' details \\ ${ }^{1}$ Inserm UMR-S839, Paris, France. ${ }^{2}$ Hopital Lariboisière APHP, Paris, France. ${ }^{3}$ Sanofi, Chilly-Mazarin, France.}

Published: 13 September 2013

doi:10.1186/1750-1326-8-S1-P31

Cite this article as: Mouton-Liger et al:: Neuroprotective role of PKR in a model of neurodegeneration due to mild impairment of oxidative metabolism. Molecular Neurodegeneration 2013 8(Suppl 1):P31. 